\title{
Mode Evolution in Strongly Coupled Plasmonic Dolmens Fabricated by Templated Assembly
}

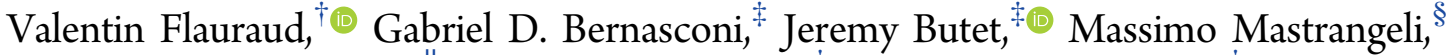 \\ Duncan T. L. Alexander, ${ }^{\| \odot}$ Olivier J. F. Martin, ${ }^{\ddagger}$ and Jürgen Brugger*, ${ }^{*}{ }^{\dagger}$ \\ ${ }^{\dagger}$ Microsystems Laboratory, ${ }^{\ddagger}$ Nanophotonics and Metrology Laboratory, and "Interdisciplinary Center for Electron Microscopy \\ (CIME), École Polytechnique Fédérale de Lausanne, 1015 Lausanne, Switzerland \\ ${ }^{\S}$ Physical Intelligence Department, Max Planck Institute for Intelligent Systems, 70569 Stuttgart, Germany
}

\section{Supporting Information}

ABSTRACT: Plasmonic antennas have enabled a wealth of applications that exploit tailored near-fields and radiative properties, further endowed by the bespoke interactions of multiple resonant building blocks. Specifically, when the interparticle distances are reduced to a few nanometers, coupling may be greatly enhanced leading to ultimate nearfield intensities and confinement along with a large energy splitting of resonant modes. While this concept is well-known, the fabrication and characterization of suitable multimers with controlled geometries and few-nanometer gaps remains highly challenging. In this article, we present the topographically templated assembly of single-crystal colloidal gold nanorods

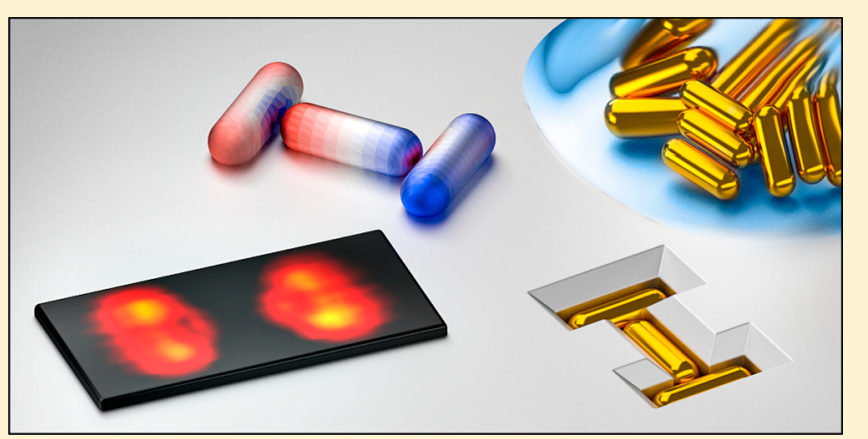
into trimers, with a dolmen geometry. This fabrication method enables the precise positioning of high-quality nanorods, with gaps as small as $1.5 \mathrm{~nm}$, which permits a gradual and controlled symmetry breaking by tuning the arrangement of these strongly coupled nanostructures. To characterize the fabricated structures, we perform electron energy loss spectroscopy (EELS) nearfield hyperspectral imaging and geometrically accurate EELS, plane wave, and eigenmode full-wave computations to reveal the principles governing the electromagnetic response of such nanostructures that have been extensively studied under plane wave excitation for their Fano resonant properties. These experiments track the evolution of the multipolar interactions with high accuracy as the antenna geometry varies. Our results provide new insights in strongly coupled single-crystal building blocks and open news opportunities for the design and fabrication of plasmonic systems.

KEYWORDS: capillary assembly, nanoparticles, nanoantennas, electron energy loss spectroscopy, eigenmodes, plasmonic dolmen

$\mathrm{P}$ lasmonic nanoantennas are well established tools for the manipulation of light at the nanoscale and for the control of light-matter interaction via the excitation of localized plasmon resonances in metallic nanostructures. ${ }^{1}$ With continuous efforts carried out for the development of novel nanofabrication methods, along with the emergence of powerful numerical simulation techniques and models, ${ }^{2}$ optical antennas have been tailored to exploit their strong scattering and absorption cross sections as well as their deep subwavelength focusing ability. ${ }^{3}$ Besides the intrinsic plasmonic properties of individual metal nanoparticles, many applications take advantage of the coupling between multiple plasmonic constituents. Indeed, the spectral tuning of different antenna building blocks and their controlled spatial arrangement enable, a wealth of unique properties such as nanometric mode confinement, ${ }^{4}$ directional color routing, ${ }^{5}$ and Fano line shapes. ${ }^{6}$

These effects, all based on the interaction between multiple plasmonic modes, are highly dependent on the coupling strength. Several coupling regimes have been identified. ${ }^{7}$ Weak coupling induces only slight shifts of the modes energies, in comparison with that of the uncoupled systems. The tailored spectral overlap and phase of the different eigenmodes may nonetheless give rise to pronounced far-field interferences. ${ }^{8}$ Moderate coupling has been thoroughly explored, due to the relative ease of fabricating nanostructures with nanogaps larger than $\sim 10 \mathrm{~nm}$. By reducing gap size further to a few nanometers, extreme coupling, and hybridization of the eigenmodes are induced, leading to large spectral splitting and ultimate nearfield intensities. 9,10

In this article, we investigate the influence of the coupling strength on the mode evolution of tunable nanorod dolmens. These trimer structures are composed of a pair of parallel nanorods hosting a third central one, perpendicular to them (Figure 1a). Dolmens have been widely investigated often considering optical excitations, in both 2D and 3D designs with mostly large interparticle gaps, since they support Fano resonances, ${ }^{11}$ which are advantageous for the fabrication of plasmonic sensors $^{12-14}$ and plasmonic nanorulers, ${ }^{15}$ as well as

Received: December 22, 2016

Published: May 22, 2017 
a

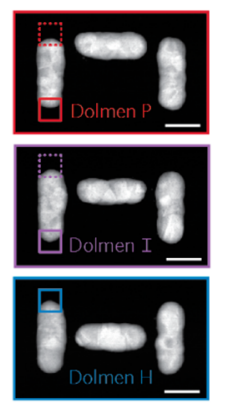

Experimental EELS maps

c
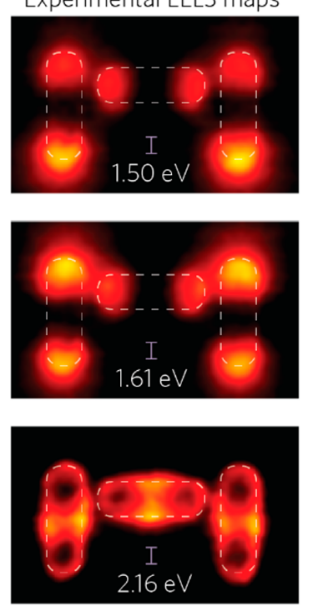

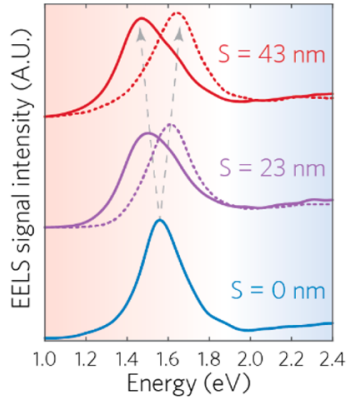

Simulated eigenmodes
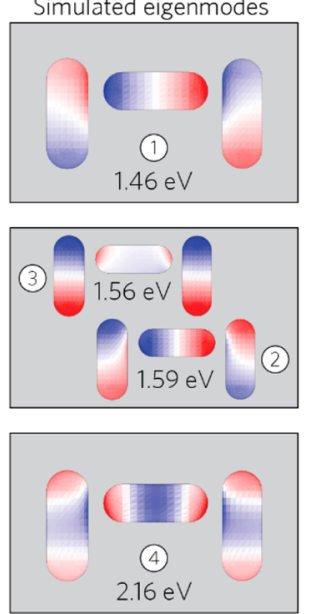
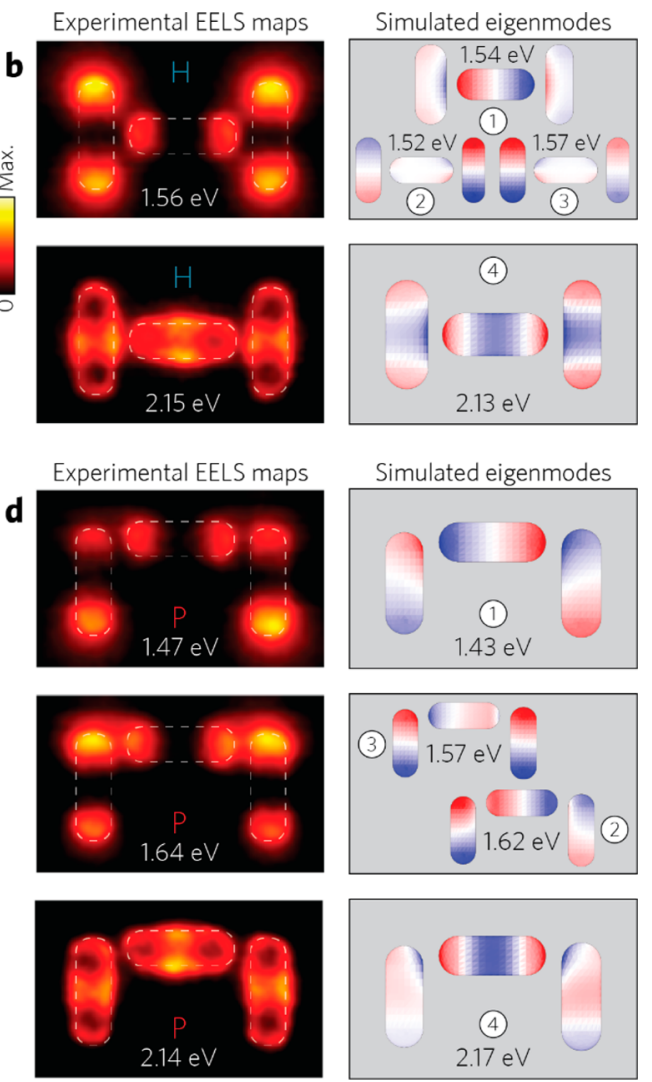

Figure 1. EELS analysis of weakly coupled Au dolmens fabricated by electron beam lithography and thin-film patterning. (a) High angle annular dark field scanning transmission electron microscope (HAADF-STEM) images of three different dolmens with varying offset parameter $S$ for the central horizontal nanorods and corresponding experimental EEL spectra extracted from the regions highlighted in the HAADF images. (b) Experimental EELS maps and corresponding simulated eigenmodes for the symmetric nanodolmen $H(S=0)$. (c, d) Similar analysis for the nanodolmen $I(S=23$ $\mathrm{nm})$, and the nanodolmen $P,(S=43 \mathrm{~nm})$. All scale bars are $50 \mathrm{~nm}$. Each EELS map is displayed with a colorscale normalized to its maximal energy loss probability.

for the observation of plasmon induced transparency ${ }^{16}$ and absorption. ${ }^{17}$ The richness of this system arises from the interplay between bright and dark modes and from a spectral response deeply related to the intricate geometric details. Beyond the implementations proposed above, such features may be enriched through the enhanced coupling and hybridization between high order modes. The latter suggests new opportunities for the design of plasmonic nanostructures, which are however practically challenged by the demanding requirements of nanometer interparticle gap fabrication.

Indeed, the fabrication of highly coupled systems with a fully controlled geometry is still elusive to date. Several lithographic attempts for the fabrication of nanometer scale gaps have been demonstrated for simple geometries such as dimers ${ }^{18}$ and bowties, ${ }^{19}$ but line edge roughness, repeatability, and scalability remain challenging to control. An efficient approach relies on the manipulation of colloids to couple them in pairs and multimers $^{20}$ or by placing them above a metallic film. ${ }^{21}$ Prompted by the vast library of anisotropic building blocks readily available, ${ }^{22}$ surface chemistry and DNA origami ${ }^{23}$ have successfully been employed to provide further levels of colloidal organization and functionality in static $^{24}$ and dynamic configurations. ${ }^{25,26}$ Such techniques provide a simple access to single or few nanometer interparticle distances often defined by molecular spacers. ${ }^{27,28}$

In the present work, we utilize a templated colloidal assembly method to fabricate highly plasmonic coupled systems. This method combines at once the intrinsic crystalline and geometric qualities of chemically synthesized nanoparticles, the simple definition of gaps by molecular elements, and the patterning versatility of nanolithography. Using this approach, and in opposition to colloid surface functionalization alone, whereby energy minima define a limited set of assembly configuration, $^{29,30}$ we have recently demonstrated highly accurate nanorod positioning in predetermined patterns with arbitrary geometries by capillary assembly. ${ }^{31}$ These are essential prerequisites for the fabrication of complex plasmonic nanostructures relying on colloidal building blocks. Here we extend this concept by performing a finely tunable assembly of multiple anisotropic building blocks within a single trap. This represents, to the best of our knowledge, the first fully deterministic assembly of such complex structures, controlling both relative and absolute position of three nanorods with gap distances of only a few nanometers.

To probe the electromagnetic response of coupled dolmens with various geometric configurations, and to assess the quality of the fabricated structures, electron energy loss spectroscopy (EELS) characterization was performed in a scanning transmission electron microscope. While both scanning near-field optical microscopy $^{32}$ and photoemission electron microscopy ${ }^{33}$ have been used to image the near-field properties of multimeric structures, EELS in modern monochromated instruments is an ideal method for the mapping of plasmonic resonances with ultimate spatial and high spectral resolution. ${ }^{34}$ Due to the 


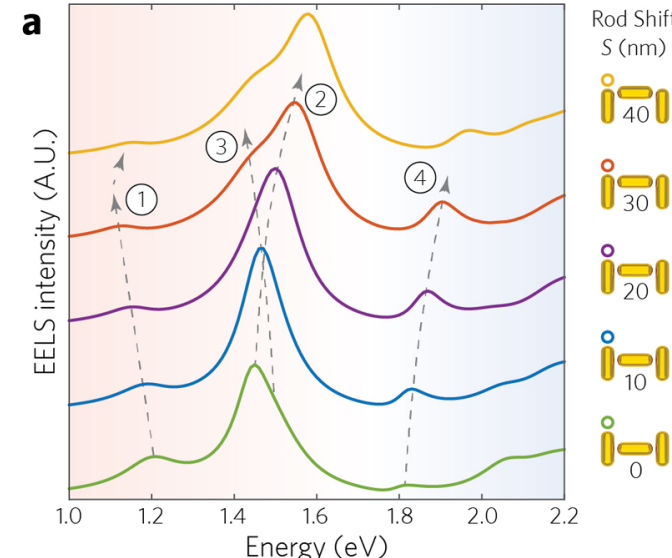

C

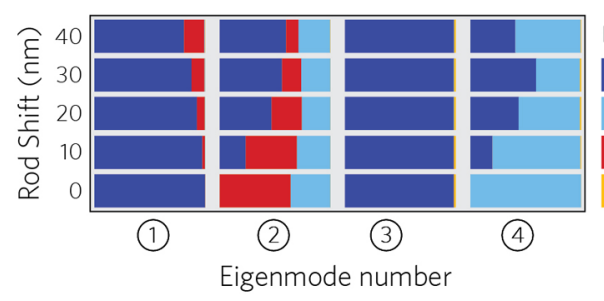

b

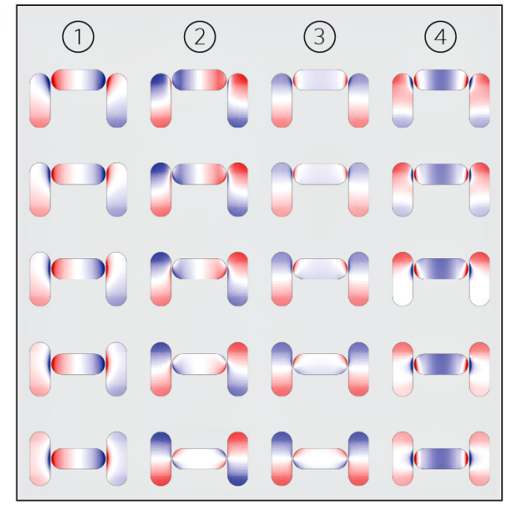

d

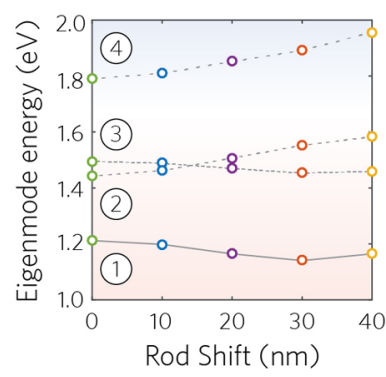

Figure 2. EELS simulations of symmetric dolmens with $2 \mathrm{~nm}$ gaps. (a) EEL spectra simulated for ideal dolmen geometries with five different central nanorod offset parameters $S$ from 0 to $40 \mathrm{~nm}$. All nanorods are $40 \mathrm{~nm} \times 105 \mathrm{~nm}$ and are separated horizontally by a $2 \mathrm{~nm}$ gap. The impact parameters for the EEL spectra are indicated on the schematics of the dolmen structures. Four main resonances numbered from (1) to (4) are highlighted. (b) 20 eigencharges computed from the eigenmodes corresponding to the labeled resonances in the EEL spectra. Eigencharges colormaps were saturated to allow a clear representation of the modes when the charges were highly confined in the nanogap regions. (c) Multipolar decomposition of the four identified modes for each of the five geometries. Each bar represents the total scattering decomposed in the different contributions. (d) Eigenmode spectral positions as functions of the central nanorod offset parameter $S$.
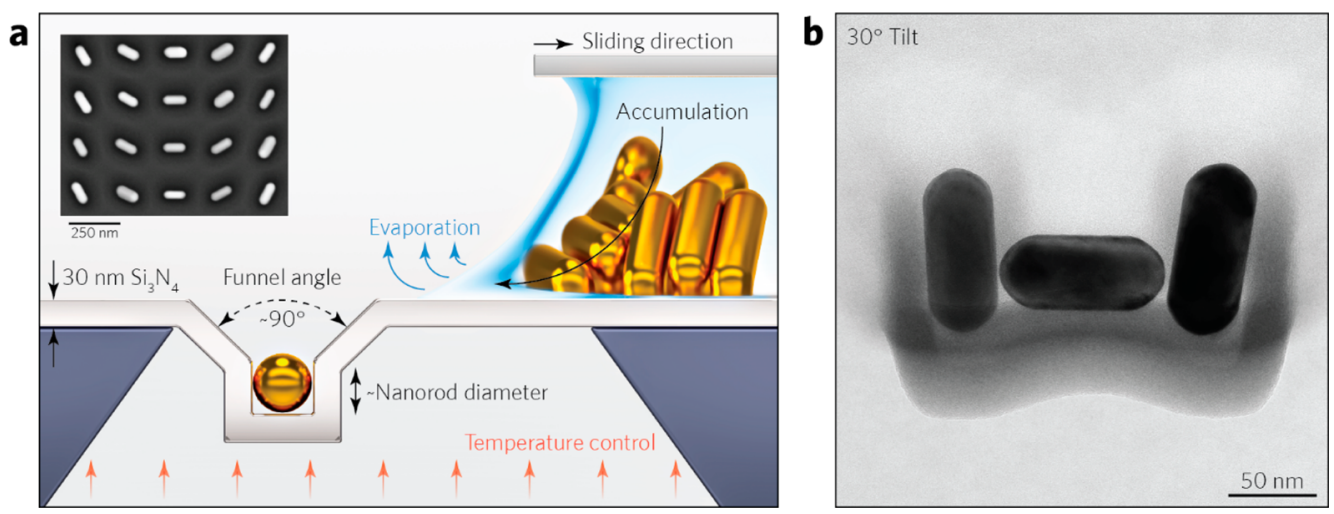

Figure 3. Capillary assembly process for the fabrication of strongly coupled Au dolmens based on single crystal nanorods. (a) Schematic representation of the capillary assembly process. Funneled traps patterned in a $30 \mathrm{~nm}$ thick silicon nitride membrane selectively and tightly capture the nanorods from the solution into predetermined locations on the substrate. The designed funnel allows for a deterministic nanorod placement and orientation as highlighted in the SEM micrograph of the inset, here on a solid silicon substrate. (b) Tilted view transmission electron image of an assembled dolmen structure.

nanometric localization of the electron probe and its intrinsic field symmetry, in addition to its sensitivity to both the radiative and nonradiative decay channels, bright modes and dark ones (with a vanishing dipole moment) are efficiently imaged. $^{35-38}$ The cylindrical symmetry of the probe also implies that asymmetric charge distributions in nanogap regions are inefficiently excited. ${ }^{39}$ Typically, bonding modes associated with intense hotspots are not revealed in gap regions, although these modes can be probed efficiently at other locations in the structures. This distinction can be exploited to interpret the nature of the different modes in addition to measuring their inherent spectral and spatial specificities. As EELS allows direct insight into the modal nature of the system, which defines a base for its response independent of excitation conditions, here the identification of the underlying coupling mechanisms of dolmen nanostructures is complemented by a rigorous and geometry accurate full wave numerical eigenmode analysis ${ }^{40}$ besides the simulation of the EELS spectra. By performing these experimental and computational investigations under different gap and geometric conditions at once, the nature and the highly geometry sensitive response of strongly coupled systems is evidenced. 
The article is organized as follows. The case of dolmens made by lithography with relatively large gaps is considered first, in order to address the plasmon coupling in the moderate regime. In a second part, the plasmon coupling in ideal dolmens made of gold nanorods with the same dimensions separated by $2 \mathrm{~nm}$ gaps is numerically investigated, allowing to discuss the mode evolutions in the strong coupling regime. Finally, gold dolmens with small gaps fabricated with capillary assembly are considered. In this last section, the experimental data are supported by simulations done considering the experimentally measured rod geometries, including the symmetry breaking induced by the size dispersion.

\section{RESULTS AND DISCUSSION}

Dolmens Made by Lithography: The Moderate Coupling Regime. Prior to the investigation of strongly coupled structures, a baseline of interactions in the moderate coupling regime is presented. Although moderately coupled structures may also be produced by assembly, Au dolmens are first fabricated by electron beam lithography (EBL) and lift-off. This is meant to provide a direct comparison of the novel fabrication method with a benchmarked standard. PMMA is coated and exposed on freestanding $30 \mathrm{~nm}$ thick $\mathrm{Si}_{3} \mathrm{~N}_{4}$ membranes guaranteeing a lithographic resolution mostly limited by forward scattering. A collection of dolmens with varying central nanorod offset is fabricated with target nanorod dimensions of $40 \mathrm{~nm} \times 105 \mathrm{~nm}$ and a gap width varying from 5 to $30 \mathrm{~nm}$ in $5 \mathrm{~nm}$ steps. After performing TEM metrology, the geometries with $15 \mathrm{~nm}$ gap size present the smallest distances between the nanorods while having noncontacted and uniform gap distributions, and are thus chosen as prototypical standards for the lithographic approach. Three dolmens with a vertical offset of the central nanorod, parameter $S$, chosen equal to 0 , 20 , and $40 \mathrm{~nm}$ are mapped and investigated by EELS (Figure $1)$. In the context of these structures, gold nanodolmens with $\pi$ shape and $20 \mathrm{~nm}$ gap distances have already been investigated using EELS. ${ }^{35,36}$

When the EELS signal is compared at each end of one of the vertical nanorods in the three dolmen structures (Figure 1a), a gradual evolution of the initial resonance peak into multiple resonances of different energy is observed as the offset of the central nanorod increases. The EELS maps and the corresponding calculated eigenmode give insights into the nature of this evolution and underlying mode coupling mechanisms. For the symmetric dolmen, labeled $H$, simulations reveal that the EELS map at the resonant energy $(1.56 \mathrm{eV})$ consists of an overlap of three eigenmodes, which are closely overlapped in energy and hence not spectrally resolvable in EELS. Mode (1) is the longitudinal bonding dipole, which can be efficiently driven by a plane wave polarized along the central nanorod main axis whereas mode (3) corresponds to the mode that can be optically driven with a polarization along the axis of the two vertical nanorods (see Figure S1 in the Supporting Information for plane wave spectra). Mode (2) is characterized by out of phase longitudinal dipoles in the vertical nanorods. For the dolmen $H$, no energy splitting between the modes is observed due to the limited near field overlap of the dipole modes supported by each nanorod. When the central nanorod is displaced by $23 \mathrm{~nm}$ (dolmen I, Figure 1c) and $43 \mathrm{~nm}$ (dolmen $P$, Figure 1d), a gradual energy splitting occurs between the longitudinal bonding mode (1) moving to lower energies and the modes (2). Now that mode (1) is spectrally separated, its spatial distribution can be isolated, with its a
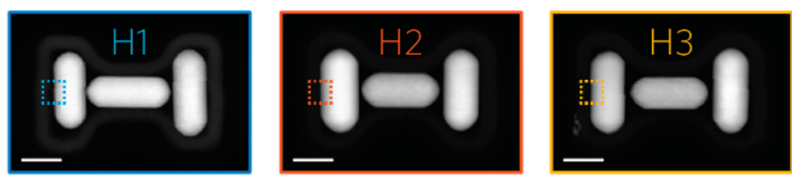

b
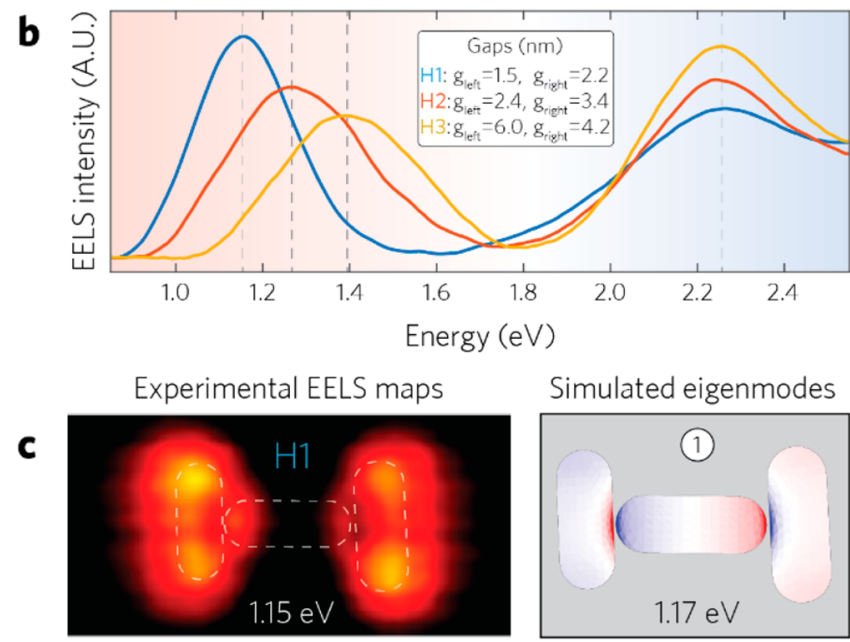

Simulated eigenmodes

d

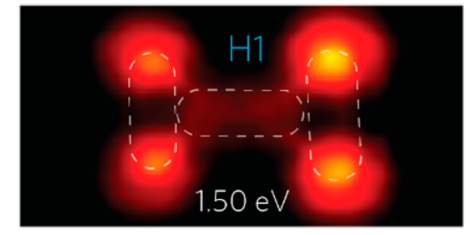

e
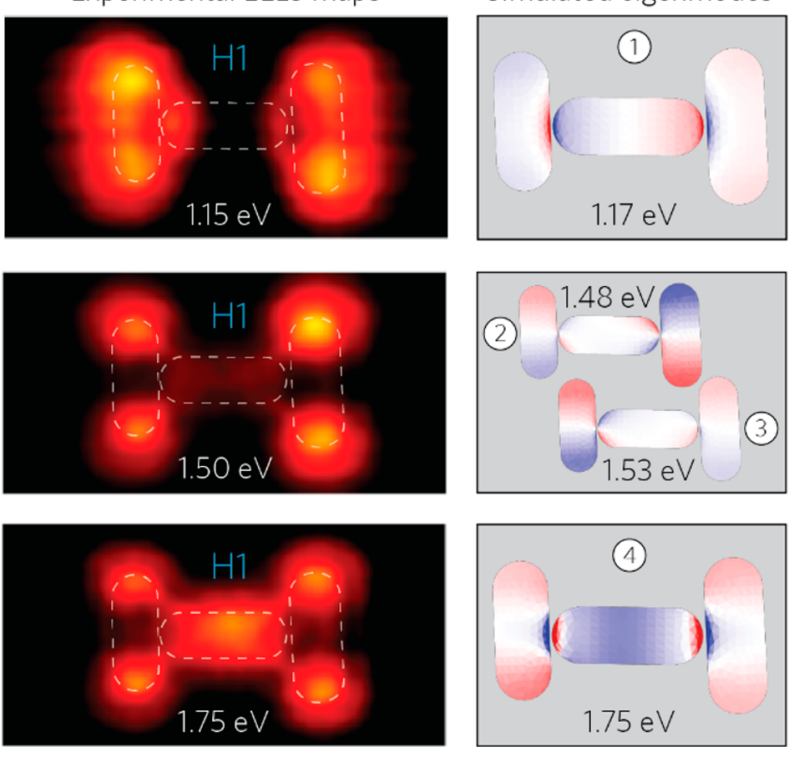

Figure 4. Gradual coupling and EELS analysis for symmetric dolmens fabricated by capillary assembly. (a) HAADF image of three quasisymmetric assembled dolmens with gaps ranging from 1.5 to $6 \mathrm{~nm}$. (b) Experimental EELS spectra extracted at the highlighted impact positions. (c-e) EELS maps end eigenmodes of dolmen H1. (c) EELS map and eigenmode associated with the low energy bonding mode. (d) EELS map at $1.50 \mathrm{eV}$ with modes (2) and (3) that are spectrally overlapped. (e) EELS map of the higher order mode (4) dominated by quadrupoles and corresponding eigencharges. Each EELS map is displayed with a colorscale normalized to its maximal energy loss probability.

bonding nature evidenced by the minimal EELS intensity in the gap. ${ }^{39}$ The excitation of mode (2), which tends toward an antibonding dipole arrangement between the nanorods as $S$ increases, is consequently responsible for the enhanced EELS intensity in the gap regions. This selective excitation of antibonding modes when the electron beam passes through the nanogaps effectively enables the distinction between modes (1) and (2), even though the energy splitting is as small as $0.11 \mathrm{eV}$ in the case of the dolmen $I$. While these lower order modes are sensitive to the central nanorod offset in this moderate coupling regime, that is not the case for the energy of higher order modes based on the interaction of quadrupolar modes in each nanorod, such as mode (4). This is inherent to the fast spatial decay of the electric field associated with high order modes, which results in weaker coupling strength than for dipole modes. Interestingly, as shown in Figure S1, the spectral 
a

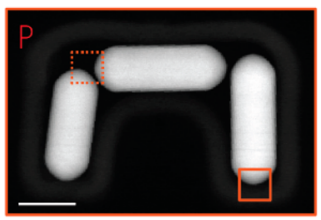

b

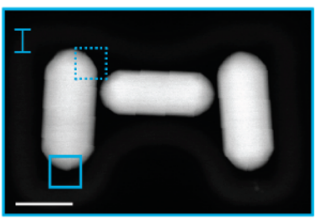

e
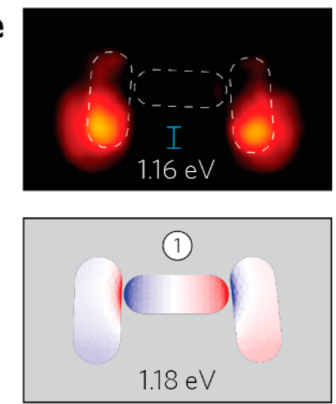

f
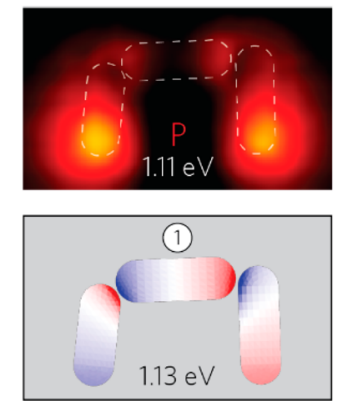

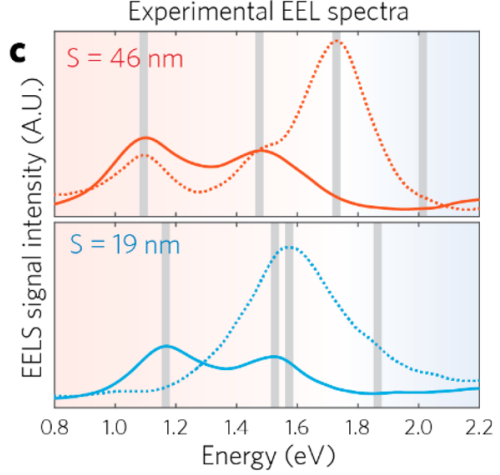

Experimental EEL spectra
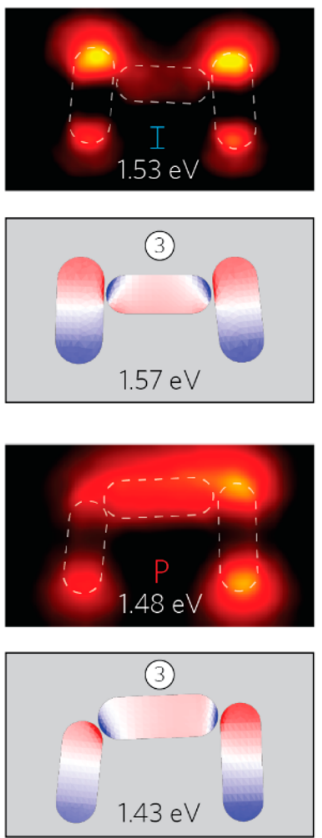
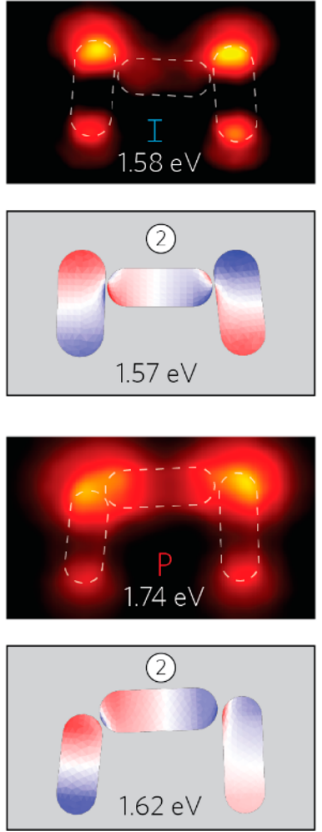

Simulated EEL spectra
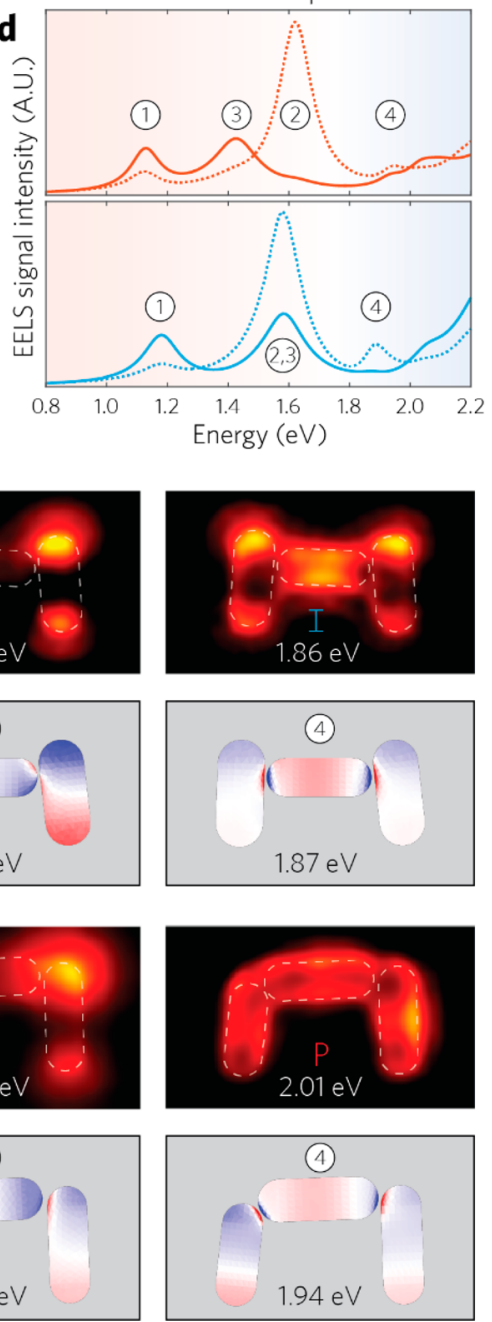

Figure 5. EELS analysis for intermediate and fully offset dolmens fabricated by capillary assembly. (a) HAADF image of an assembled dolmens with $19 \mathrm{~nm}$ central rod offset and (b) $43 \mathrm{~nm}$ offset. (c) Experimental EELS spectra extracted from the locations highlighted in the HAADF images and corresponding simulations (d). (e) EELS maps and associated eigencharges of dolmen $I$ with $19 \mathrm{~nm}$ central rod offset. (f) EELS maps and associated eigencharges of dolmen $P$ with $43 \mathrm{~nm}$ central rod offset. Each EELS map is displayed with a colorscale normalized to its maximal energy loss probability.

proximity of modes (1) and (2) leads to the gradual appearance of a Fano profile under optical illumination due to the interference between these two modes. ${ }^{41}$

Ideal Dolmens with 2 nm Gaps. To consider how behavior may differ in a stronger coupling regime, we investigate numerically ideal dolmens constituted of three similar hemispherical nanorods of $105 \mathrm{~nm}$ length and $40 \mathrm{~nm}$ diameter with $2 \mathrm{~nm}$ interparticle gaps. Five structures are considered, from the symmetric case $(S=0)$ up to a maximum vertical shift of the central nanorod $S=40 \mathrm{~nm}$. The EELS response of these dolmens is computed for two positions of the electron beam; one at the top end of one vertical nanorod (Figure 2a) and one close to the side of the central nanorod (Figure S3) in order to probe all the modes of interest. Each eigenmode is computed and shown, as a surface charge distribution, in Figure $2 \mathrm{~b}$. In this ideal symmetric geometry, modes (1) and (2) have an odd charge parity with respect to the vertical symmetry plane, whereas modes (3) and (4) exhibit an even parity. Modes (1) and (4) are respectively characterized by a longitudinal dipolar and quadrupolar charge distribution on the central nanorod, in all geometric configurations. These two modes are significantly red-shifted in comparison with the weakly coupled dolmens discussed previously; a behavior that is explained by a strong bonding interaction to the adjacent rods. In the case of mode (1), increasing the offset $S$ results in a gradual evolution of the charge distributions in the vertical nanorods, from a transverse dipolar distribution to a longitudinal dipole. This progressively allows the coupling of three longitudinal dipoles at the same energy leading to the redshift of the mode resonance. For mode (4), the inverse tendency is observed. The coupling with the adjacent nanorods is weakened by the position offset resulting in a blue shift of mode (4). Indeed, for $S=0$, the central rod quadrupolar charge distribution is stretched toward the adjacent rods and tends to the charge distribution of the longitudinal quadrupole of an isolated nanorod (Figure S2) with increasing S. For modes (2) and (3), a crossing between the resonant energies is observed as the central nanorod offset increases. This crossing indicates that modes (2) and (3) do not interact due to their symmetry properties. $^{42}$ In the case of mode (2), characterized by the two out-of-phase vertical dipoles, the charge distribution supported by the central nanorod evolves from a transverse quadrupole to 
a longitudinal dipole with an antibonding interaction with other dipoles yielding to an effective blue shift of mode (2). For mode (3), characterized by two in-phase dipoles on the vertical nanorods, the central nanorod gradually moves into the near field of the vertical dipoles, here with a bonding interaction, yielding to a red-shift of the mode energy.

Together, these observations clearly indicate that the electromagnetic response of highly coupled nanodolmens, with a $2 \mathrm{~nm}$ gap, has a multipolar nature. Namely, short gaps enable an important modulation of the multipolar response of the dolmen via a collective coupling mediated by the central nanorod position. To investigate this in detail, a multipole expansion has been performed for each eigenmode (Figure $2 c$ ), that is, the contribution of the electric and magnetic multipoles to the radiation pattern has been determined. ${ }^{43}$ The nature of mode (1) corresponds to a pure electric dipole for $S=0$, with a magnetic dipole contribution appearing as the central nanorod offset increases. Indeed, for $S=40 \mathrm{~nm}$, a current loop is observed, similar to that of a split ring resonator. ${ }^{44}$ For mode (2), the strong magnetic dipole contribution originating from the out-of-phase dipoles supported by the two vertical nanorods is gradually complemented by the appearance of an electric dipole contribution established on the central nanorod. Interestingly, when the central nanorod reaches the $S=40$ offset, the gap between nanorods jumps from 2 to $2.66 \mathrm{~nm}$, due to the rounded apex of the nanorods. This translates into a decrease of the near-field coupling and a resultant blue shift (Figure $2 \mathrm{~d}$ ) of mode (1). Overall, therefore, gaps in the few nanometers range in the context of dolmen structures present a 2 -fold interest. First, both the spectral tunability and geometry-based mode energy shift sensitivity are enhanced. Second, important contributions of quadrupoles and transverse dipoles are enabled. This results in important redistributions of the charges as the geometry evolves, that is, as the central nanorod offset changes.

Strongly Coupled Dolmens Made with Capillary Assembly. To investigate these possibilities experimentally, dolmens with nanometer scale gaps have been fabricated by capillary assembly. In the process, a drop of colloidal solution containing $\mathrm{Au}$ nanorods is heated and swept across a topographically patterned template (see schematic in Figure 3a). As previously reported, ${ }^{31}$ upon heating, the nanorods tightly accumulate at the contact line of the drop, enter the topographic traps when crossing them, and finally dry into a final location with deterministic position and orientation (inset Figure $3 a$ ). In order to obtain the desired trap funneled profile, a critical parameter for an efficient nanorod assembly, templates are fabricated as follows. Holes are first etched into silicon substrates by the transfer of resist tapering into the silicon to produce a straight trap decorated by an upper funnel that increases the nanorod capture cross-section (see Supporting Information for details). The silicon substrates are then coated by $30 \mathrm{~nm}$ of low-stress $\mathrm{Si}$ rich $\mathrm{Si}_{x} \mathrm{~N}_{y}$ by low pressure chemical vapor deposition that is subsequently released from the backside by potassium hydroxide $\mathrm{Si}$ wet etching. After functionalization with a hydrophobic silane, the substrates are cleaved into $17 \times 17 \mathrm{~mm}^{2}$ dies for capillary assembly and subsequently cleaved into $3 \times 3 \mathrm{~mm}^{2} \mathrm{~mm}$ samples with single $\mathrm{Si}_{x} \mathrm{~N}_{y}$ windows for TEM measurements. A typical dolmen structure is shown in Figure $3 \mathrm{~b}$, underlining that interparticle distances as short as $1.5 \mathrm{~nm}$ can indeed be achieved. In addition to nanometric interparticle distances, capillary assembly harnesses the plasmonic qualities of single crystalline gold, ${ }^{45}$ giving a similar design versatility as previously demonstrated by the FIB milling of single crystal gold flakes. ${ }^{4,47}$

Among all the fabricated nanostructures, symmetric dolmens (denoted $\mathrm{H} 1, \mathrm{H} 2$, and $\mathrm{H} 3$ ) with gap sizes between 1.5 and 6 $\mathrm{nm}$ are studied first (Figure 4a). As shown from the previous simulations and measurements, the lowest energy bonding mode (1) is the most sensitive to gap variations within the structure. This is experimentally demonstrated by integrating the EELS signal along the side of the dolmens $\mathrm{H} 1, \mathrm{H} 2$, and $\mathrm{H} 3$ (Figure $4 \mathrm{~b}$ ). The energy of mode (1) gradually shifts from 1.4 to $1.15 \mathrm{eV}$ as the gaps are reduced, in good agreement with the expected shift calculated for the simulated dolmens. As mentioned previously, modes (2) and (3) spectrally overlap for symmetric dolmens. Although these two modes cannot be resolved directly in EELS because of their overlap, a slight asymmetry of the structure $\mathrm{H} 1$ (with the smallest gap sizes) appears to yield a stronger charge localization on the rightmost nanorod. This is well shown in its EELS map, in accordance with the computed eigenmode (Figure 4d). Mode (4) also exhibits a strong red-shift in comparison with the $\sim 15 \mathrm{~nm}$ gap lithographic nanodolmens, as predicted by simulations. Further comparing to mode (4) in the lithographic structures (Figure 1), in $\mathrm{H} 1$ we also observe the disappearance of the two dark regions close to the central nanorod extremities, which correspond to nodes in the charge distributions. Additionally, strong EELS signal is measured in the central nanorod (Figure $4 \mathrm{e})$. These observations are direct consequences of the stretching of the central quadrupole via the coupling to the adjacent nanorods.

Upon a shift of the central nanorod, a splitting of modes (1) and (4) is expected along with the crossing of modes (2) and (3) (Figure 2). To study this, we finally consider two assembled dolmens with central nanorod offset of 19 and $46 \mathrm{~nm}$ and average gap sizes of $2.5 \mathrm{~nm}$ (Figure 5a,b). Following the methodology described above, the EELS signal is extracted from the bottom end of one of the vertical nanorods along with the EELS signal from a gap region for both nanostructures. The comparison between experimental (Figure 5c) and simulated (Figure 5d) spectra reveals an excellent spectral agreement and broader resonances for experimental data are primarily due to the convolution with the EELS zero-loss peak of $0.11 \mathrm{eV}$ fwhm. For both dolmens, the low energy bonding mode (1) is gradually red-shifted to 1.16 and $1.11 \mathrm{eV}$ for $S=19 \mathrm{~nm}$ and $S=46 \mathrm{~nm}$, respectively. The same mode was measured at $1.26 \mathrm{eV}$ for the symmetric dolmen $\mathrm{H} 2$ with similar gap dimensions. For the intermediate dolmen $I$ made by capillary assembly (Figure 5e), modes (2) and (3) are not distinguished experimentally owing to a spectral overlap revealed by the simulations. In comparison, these modes are well distinguished for a large offset of the central nanorod (Figure 5f), as evidenced in both the EELS spectra and the EELS maps. The energy difference between the modes (1) and (4), which increases from 0.7 to $0.9 \mathrm{eV}$, is another useful metric demonstrating the control of the assembly and sensitivity of the displacement of the central nanorod in this highly coupled system.

Remarkably, the capillary-assembled nanorod structures are stable even under prolonged electron beam irradiation, also when they are separated by the smallest possible interparticle distance as determined by the collapsed cetyltrimethylammonium bromide (CTAB) surfactant bilayer $(1.5 \mathrm{~nm})$. By instead fusing neighboring nanorods using an oxygen plasma treatment and annealing, the lower energy modes are red-shifted to energies below $1 \mathrm{eV}$, indicating the formation of charge transfer 
plasmons $^{31,48}$ (Figure S4). This hints to a unique alternative to Au flake milling for the fabrication of monomeric antennas with single crystal building blocks. ${ }^{46,47}$ Additionally, we have also investigated the influence of asymmetries for a dolmen in which a vertical nanorod is significantly longer than the other ones while having zero offset for the shorter vertical nanorod respectively to the horizontal one (Figure S5). These measurements allow us to highlight the significant impact of asymmetry on near field localization and energy splitting of modes (2) and (3), which respectively support out-of-phase and in-phase longitudinal dipoles on the vertical nanorods. In the case of this asymmetric dolmen, modes (2) and (3) become strongly localized on the longer and shorter nanorods, respectively, and markedly split in energy. Owing to the different net dipole moments in each vertical nanorod, modified modes (2) and (3) are expected to present a radiative nature, providing a good metric for the far-field analysis of the dolmen symmetry disentangled from offset parameter and gap width.

\section{CONCLUSION}

In conclusion, we have experimentally and theoretically investigated the near field coupling mechanisms in gold nanodolmens with different arrangements and interparticle distances. By using capillary assembly, interparticle gaps as short as $1.5 \mathrm{~nm}$ were produced leading to enhanced coupling and large energy splitting between bonding and antibonding modes. Additionally, the short gap distances produce a strong contribution from high energy quadrupole and transverse dipole modes. Their influence on the electromagnetic response were tracked by a gradual symmetry breaking of the nanodolmen via controlled vertical offsetting of the central nanorod. By relating the experimental findings to eigenmode simulations and multipolar decompositions, an excellent agreement was found between the response of idealized and fabricated structures. This work therefore paves the way for the fabrication of complex plasmonic nanoparticle assemblies that may enable new opportunities for the design of plasmonic rulers, refractive index sensors, and nonlinear antennas based on single crystal, low loss building blocks. Furthermore, and although our experiments have been fully interpreted and simulated relying on classical theory, combining the capillary assembly method with specific molecular spacers is a promising way to control plasmon resonances with molecular tunnel junction. $^{49}$

\section{ASSOCIATED CONTENT}

\section{S Supporting Information}

The Supporting Information is available free of charge on the ACS Publications website at DOI: 10.1021/acsphotonics.6b01026.

Plane wave excitation, hybridization pattern, supplementary EELS spectra, fused nanorods, and asymmetric assemblies (PDF).

\section{AUTHOR INFORMATION}

\section{Corresponding Author}

*E-mail: juergen.brugger@epfl.ch.

ORCID ${ }^{\circ}$

Valentin Flauraud: 0000-0002-1393-3198

Jeremy Butet: 0000-0001-9598-9074

Duncan T. L. Alexander: 0000-0003-4350-8587
Olivier J. F. Martin: 0000-0002-9574-3119

Jürgen Brugger: 0000-0002-7710-5930

\section{Notes}

The authors declare no competing financial interest.

\section{ACKNOWLEDGMENTS}

The research leading to these results has received funding from the European Commission's Seventh Framework Programme (FP7-ICT-2011-7) under Grant Agreements 288263 (NanoVista) and ERC-2015-AdG-695206 (Nanofactory) and the Swiss National Science Foundation (SNSF; 200020_153662). The authors gratefully acknowledge the valuable support from the EPFL center of micro- and nanofabrication (CMi).

\section{REFERENCES}

(1) Maier, S. A. Plasmonics: Fundamentals and Applications; Springer Science + Business Media LLC: New York, 2007.

(2) Gallinet, B.; Butet, J.; Martin, O. J. F. Numerical methods for nanophotonics: standard problems and future challenges. Laser Photonics Rev. 2015, 9, 577-603.

(3) Paolo, B.; Jer-Shing, H.; Bert, H. Nanoantennas for visible and infrared radiation. Rep. Prog. Phys. 2012, 75, 024402.

(4) Chikkaraddy, R.; de Nijs, B.; Benz, F.; Barrow, S. J.; Scherman, O. A.; Rosta, E.; Demetriadou, A.; Fox, P.; Hess, O.; Baumberg, J. J. Single-molecule strong coupling at room temperature in plasmonic nanocavities. Nature 2016, 535, 127-130.

(5) Shegai, T.; Chen, S.; Miljković, V. D.; Zengin, G.; Johansson, P.; Käll, M. A bimetallic nanoantenna for directional colour routing. Nat. Commun. 2011, 2, 481.

(6) Verellen, N.; Sonnefraud, Y.; Sobhani, H.; Hao, F.; Moshchalkov, V. V.; Van Dorpe, P.; Nordlander, P.; Maier, S. A. Fano Resonances in Individual Coherent Plasmonic Nanocavities. Nano Lett. 2009, 9, 1663-1667.

(7) Prodan, E.; Radloff, C.; Halas, N. J.; Nordlander, P. A hybridization model for the plasmon response of complex nanostructures. Science 2003, 302, 419-422.

(8) Luk'yanchuk, B.; Zheludev, N. I.; Maier, S. A.; Halas, N. J.; Nordlander, P.; Giessen, H.; Chong, C. T. The Fano resonance in plasmonic nanostructures and metamaterials. Nat. Mater. 2010, 9, $707-715$

(9) Halas, N. J.; Lal, S.; Chang, W. S.; Link, S.; Nordlander, P. Plasmons in strongly coupled metallic nanostructures. Chem. Rev. 2011, 111, 3913-61.

(10) Kauranen, M.; Zayats, A. V. Nonlinear plasmonics. Nat. Photonics 2012, 6, 737-748.

(11) Yan, C.; Martin, O. J. F. Periodicity-Induced Symmetry Breaking in a Fano Lattice: Hybridization and Tight-Binding Regimes. ACS Nano 2014, 8, 11860-11868.

(12) Gallinet, B.; Martin, O. J. Refractive index sensing with subradiant modes: a framework to reduce losses in plasmonic nanostructures. ACS Nano 2013, 7, 6978-87.

(13) Gallinet, B.; Siegfried, T.; Sigg, H.; Nordlander, P.; Martin, O. J. Plasmonic radiance: probing structure at the Angstrom scale with visible light. Nano Lett. 2013, 13, 497-503.

(14) Butet, J.; Martin, O. J. Refractive index sensing with Fano resonant plasmonic nanostructures: a symmetry based nonlinear approach. Nanoscale 2014, 6, 15262-70.

(15) Liu, N.; Hentschel, M.; Weiss, T.; Alivisatos, A. P.; Giessen, H. Three-Dimensional Plasmon Rulers. Science 2011, 332, 1407-1410.

(16) Liu, N.; Langguth, L.; Weiss, T.; Kastel, J.; Fleischhauer, M.; Pfau, T.; Giessen, H. Plasmonic analogue of electromagnetically induced transparency at the Drude damping limit. Nat. Mater. 2009, 8, $758-762$.

(17) Taubert, R.; Hentschel, M.; Kästel, J.; Giessen, H. Classical Analog of Electromagnetically Induced Absorption in Plasmonics. Nano Lett. 2012, 12, 1367-1371. 
(18) Zhu, W.; Crozier, K. B. Quantum mechanical limit to plasmonic enhancement as observed by surface-enhanced Raman scattering. Nat. Commun. 2014, 5, 5228.

(19) Duan, H.; Fernández-Domínguez, A. I.; Bosman, M.; Maier, S. A.; Yang, J. K. W. Nanoplasmonics: Classical down to the Nanometer Scale. Nano Lett. 2012, 12, 1683-1689.

(20) Punj, D.; Regmi, R.; Devilez, A.; Plauchu, R.; Moparthi, S. B.; Stout, B.; Bonod, N.; Rigneault, H.; Wenger, J. Self-Assembled Nanoparticle Dimer Antennas for Plasmonic-Enhanced SingleMolecule Fluorescence Detection at Micromolar Concentrations. ACS Photonics 2015, 2, 1099-1107.

(21) Hu, M.; Ghoshal, A.; Marquez, M.; Kik, P. G. Single Particle Spectroscopy Study of Metal-Film-Induced Tuning of Silver Nanoparticle Plasmon Resonances. J. Phys. Chem. C 2010, 114, 7509-7514.

(22) Sau, T. K.; Rogach, A. L. Nonspherical noble metal nanoparticles: colloid-chemical synthesis and morphology control. Adv. Mater. 2010, 22, 1781-804.

(23) Tan, S. J.; Campolongo, M. J.; Luo, D.; Cheng, W. Building plasmonic nanostructures with DNA. Nat. Nanotechnol. 2011, 6, 268276.

(24) Acuna, G. P.; Möller, F. M.; Holzmeister, P.; Beater, S.; Lalkens, B.; Tinnefeld, P. Fluorescence Enhancement at Docking Sites of DNADirected Self-Assembled Nanoantennas. Science 2012, 338, 506-510.

(25) Kuzyk, A.; Schreiber, R.; Zhang, H.; Govorov, A. O.; Liedl, T.; Liu, N. Reconfigurable 3D plasmonic metamolecules. Nat. Mater. 2014, 13, 862-866.

(26) Yang, S.; Ni, X. J.; Yin, X. B.; Kante, B.; Zhang, P.; Zhu, J.; Wang, Y.; Zhang, X. Feedback-driven self-assembly of symmetrybreaking optical metamaterials in solution. Nat. Nanotechnol. 2014, 9, $1002-1006$.

(27) Ciracì, C.; Hill, R. T.; Mock, J. J.; Urzhumov, Y.; FernándezDomínguez, A. I.; Maier, S. A.; Pendry, J. B.; Chilkoti, A.; Smith, D. R. Probing the Ultimate Limits of Plasmonic Enhancement. Science 2012, 337, 1072-1074.

(28) Bidault, S.; Devilez, A.; Maillard, V.; Lermusiaux, L.; Guigner, J.M.; Bonod, N.; Wenger, J. Picosecond Lifetimes with High Quantum Yields from Single-Photon-Emitting Colloidal Nanostructures at Room Temperature. ACS Nano 2016, 10, 4806-4815.

(29) Gao, B.; Arya, G.; Tao, A. R. Self-orienting nanocubes for the assembly of plasmonic nanojunctions. Nat. Nanotechnol. 2012, 7, 433437.

(30) Jones, S. T.; Taylor, R. W.; Esteban, R.; Abo-Hamed, E. K.; Bomans, P. H.; Sommerdijk, N. A.; Aizpurua, J.; Baumberg, J. J.; Scherman, O. A. Gold nanorods with sub-nanometer separation using cucurbit[n] uril for SERS applications. Small 2014, 10, 4298-303.

(31) Flauraud, V.; Mastrangeli, M.; Bernasconi, G. D.; Butet, J.; Alexander, D. T. L.; Shahrabi, E.; Martin, O. J. F.; Brugger, J. Nanoscale topographical control of capillary assembly of nanoparticles. Nat. Nanotechnol. 2016, 12, 73-80.

(32) Khunsin, W.; Dorfmuller, J.; Esslinger, M.; Vogelgesang, R.; Rockstuhl, C.; Etrich, C.; Kern, K. Quantitative and Direct Near-Field Analysis of Plasmonic-Induced Transparency and the Observation of a Plasmonic Breathing Mode. ACS Nano 2016, 10, 2214-24.

(33) Yu, H.; Sun, Q.; Ueno, K.; Oshikiri, T.; Kubo, A.; Matsuo, Y.; Misawa, H. Exploring Coupled Plasmonic Nanostructures in the Near Field by Photoemission Electron Microscopy. ACS Nano 2016, 10, 10373-10381.

(34) Bellido, E. P.; Bicket, I. C.; McNeil, J.; Botton, G. A. Very High Resolution Energy Loss Spectroscopy: Applications in Plasmonics. Microsc. Microanal. 2016, 22, 974-975.

(35) Coenen, T.; Schoen, D. T.; Mann, S. A.; Rodriguez, S. R.; Brenny, B. J.; Polman, A.; Brongersma, M. L. Nanoscale Spatial Coherent Control over the Modal Excitation of a Coupled Plasmonic Resonator System. Nano Lett. 2015, 15, 7666-70.

(36) Coenen, T.; Schoen, D. T.; Brenny, B. J. M.; Polman, A.; Brongersma, M. L. Combined electron energy-loss and cathodoluminescence spectroscopy on individual and composite plasmonic nanostructures. Phys. Rev. B: Condens. Matter Mater. Phys. 2016, 93, 195429.
(37) Losquin, A.; Zagonel, L. F.; Myroshnychenko, V.; RodríguezGonzález, B.; Tencé, M.; Scarabelli, L.; Förstner, J.; Liz-Marzán, L. M.; García de Abajo, F. J.; Stéphan, O.; Kociak, M. Unveiling Nanometer Scale Extinction and Scattering Phenomena through Combined Electron Energy Loss Spectroscopy and Cathodoluminescence Measurements. Nano Lett. 2015, 15, 1229-1237.

(38) Bernasconi, G. D.; Butet, J.; Flauraud, V.; Alexander, D.; Brugger, J.; Martin, O. J. F. Where Does Energy Go in Electron Energy Loss Spectroscopy of Nanostructures? ACS Photonics 2017, 4, 156164.

(39) Hohenester, U.; Ditlbacher, H.; Krenn, J. R. Electron-EnergyLoss Spectra of Plasmonic Nanoparticles. Phys. Rev. Lett. 2009, 103, 106801.

(40) Bernasconi, G. D.; Butet, J.; Martin, O. J. F. Mode analysis of second-harmonic generation in plasmonic nanostructures. J. Opt. Soc. Am. B 2016, 33, 768-779.

(41) Gallinet, B.; Martin, O. J. F. Relation between near-field and far-field properties of plasmonic Fano resonances. Opt. Express 2011, 19, 22167-22175.

(42) Nordlander, P.; Oubre, C.; Prodan, E.; Li, K.; Stockman, M. I. Plasmon Hybridization in Nanoparticle Dimers. Nano Lett. 2004, 4, 899-903.

(43) Mühlig, S.; Menzel, C.; Rockstuhl, C.; Lederer, F. Multipole analysis of meta-atoms. Metamaterials 2011, 5, 64-73.

(44) Sarychev, A. K.; Shvets, G.; Shalaev, V. M. Magnetic plasmon resonance. Phys. Rev. E 2006, 73, 036609.

(45) Shao, L.; Tao, Y. T.; Ruan, Q. F.; Wang, J. F.; Lin, H. Q. Comparison of the plasmonic performances between lithographically fabricated and chemically grown gold nanorods. Phys. Chem. Chem. Phys. 2015, 17, 10861-10870.

(46) Huang, J. S.; Callegari, V.; Geisler, P.; Bruning, C.; Kern, J.; Prangsma, J. C.; Wu, X.; Feichtner, T.; Ziegler, J.; Weinmann, P.; Kamp, M.; Forchel, A.; Biagioni, P.; Sennhauser, U.; Hecht, B. Atomically flat single-crystalline gold nanostructures for plasmonic nanocircuitry. Nat. Commun. 2010, 1, 150.

(47) Celebrano, M.; Wu, X.; Baselli, M.; Großmann, S.; Biagioni, P.; Locatelli, A.; De Angelis, C.; Cerullo, G.; Osellame, R.; Hecht, B.; Duò, L.; Ciccacci, F.; Finazzi, M. Mode matching in multiresonant plasmonic nanoantennas for enhanced second harmonic generation. Nat. Nanotechnol. 2015, 10, 412-417.

(48) Pérez-González, O.; Zabala, N.; Borisov, A. G.; Halas, N. J.; Nordlander, P.; Aizpurua, J. Optical Spectroscopy of Conductive Junctions in Plasmonic Cavities. Nano Lett. 2010, 10, 3090-3095.

(49) Tan, S. F.; Wu, L.; Yang, J. K. W.; Bai, P.; Bosman, M.; Nijhuis, C. A. Quantum Plasmon Resonances Controlled by Molecular Tunnel Junctions. Science 2014, 343, 1496-1499. 